\title{
The Scale of Children's School Readiness in Poland
}

\author{
Anna Klim-Klimaszewska \\ University of Natural Science and Humanities in Siedlce, Poland
}

\begin{abstract}
The aim of the nursery school education in Poland is to support the intellectual development of children so that they were well prepared for school. That is why the Act on School Education from $19^{\text {th }}$ March 2009 states that there is one year commitment to pre-school obligation for each 5 years old child which can be fulfilled in the nursery school unit organized at primary school or in other form of nursery school education. The children who attend to nursery school institutions - no matter what their organizational formula or place is - have to execute the whole of the programme basis in order to be well prepared for school and to cope with various situations in their lives.

Thus, nursery school teachers are obliged to keep the record of their pedagogical observations systematically both with analysis of the children's behaviour thanks to which they can get to know the intellectual abilities and developmental needs of every child.

In order to check the child's school readiness in Poland there is the Pupil's School Readiness Observation Card presented in the article.
\end{abstract}

\section{Introduction}

Nursery school education is there to support the children's intellectual development so that they were well prepared for school. That is why the act on the system of education from $19^{\text {th }}$ March 2009 states that every five years old child is obliged to have one year long pre-school preparation at nursery school, nursery school unit organized at primary school or in other form of nursery school education. The children who attend the nursery school institutions - no matter what organizational formula and place they have - they need to execute the whole programme base in order to acquire the appropriate preparation to learn at school and cope with daily life situations.

The children's readiness to learn at school is an essential interdisciplinary problem of the educational practice. It is a subject of interest of developmental psychology, clinical psychology, educational and social pedagogy. For many children the moment of starting their education at school, new requirements connected with duties, the imposed environment, cognitive effort and the complexity of the social life in a group makes a significant difficulty. The research shows that first school experience is of great impact on the child's later attitude towards his job and himself and also on his achievements and the level of aspirations. The research also points out that the experience of achieving support in difficult and new situation is very meaningful both for overcoming the child's crisis and for his further development.

School makes a different environment from the ones a child has been in so far. It requires from him adjusting to the new mode of life, which is determined by systematic classes, to the imposed physical environment which is different than at home and to learn how to work in a group of peers under the guidance of an adult. A child at school has to show his resistance to physical effort and stress connected with both assessing and rivalry. He has to learn how to respect the established rules of behavior. He needs to acquire, among others, some basic abilities of reading, writing and mathematical skills.

Thus, by the school readiness it should be understood that the child achieved the level of his intellectual, emotional and social development which will enable his education at school.

The psychology of human development recognizes the time of beginning the school education as an important, prescriptive and critical period in our lives. Human developmental changes are in this conception connected with the undertaken forms of activity and with so called critical events which are of the great influence in his later life. In that particular period of life a person has to fulfill some developmental tasks, acquire some abilities and handle difficult situations so that there could take place his further development.

Starting the school education can be for a child as a critical incident. Critical incidents are characterized by the fact that they are emotionally engaging and their effects can be both close and distant in time. Whether they are positive or not depends on the child's features, his reactions on the given incident, the context of it and also on the support provided by the meaningful people.

In psychological theories of the development an important term is the developmental task. When a child starts his school education he meets new expectations from his teachers, parents, community. With the beginning of the school education there are connected the following tasks:

- gaining and using the knowledge,

- acquiring self-reliance and independence,

- taking part in the peer group life, 
- changing the dominating form of activity from play into learning,

- obtaining the ability of writing and reading.

Fulfilling by the children the developmental tasks connected with the first period of the school education is conditioned by obtaining many abilities. Gaining and acquiring the knowledge is connected with e.g. the ability to focus attention, solve problems, read and write. Together with acquiring self-reliance there is connected the ability to step around obstacles, aspire to be independent and regarding cooperation with peers - the ability to communicate and to adjust to the established agreements and rules. The process of education is connected with organizing activities on account of the aim, aspiring to achieve the goal, bearing tension.

\section{The School Readiness Scale}

The readiness to learn requires fulfilling the following conditions:

- there is an interest in a child to learn, he wants to be learned and wants to learn independently,

- there is the permanence of interest, a child continues to learn although there are some difficulties and failures,

- a child achieves progress, even the small one, while learning.

Within the term of the school readiness there is the content connected with the subjectivity, independence, a child's interests, achievements and his aspiration to overcome obstacles. This term concentrates our attention on a child and his activity. It does not divide the children into those who are mature and immature, but to those who are prepared to a various extent to be at school.

If a child starts his education without achieving the sufficient school readiness and his difficulties are not recognized and prevented then the later development of that child might be disordered. His activeness and creative attitude towards assignments might be hampered. A child may become passive and may not try to undertake the tasks connected with learning or may try to compensate for his difficulties in an adverse way. Sometimes, as soon as during the early stages of primary education, there can be seen first symptoms of typical problems, revealed together with starting school education, which are described as the results achieved below capabilities. There can also be the passive-aggressive behavior or the symptom of the learned cognitive helplessness. The child's difficulties often go with the psychosomatic disorders. The studies and experiences of the practitioners point out the close connection between failures while learning and emotional problems or social maladjustment. It is also stated that the support, which is given to children early at the beginning of the school education, has the positive influence on their later decisions and choices in their lives.

Thus, the nursery school teachers in Poland are obliged to lead pedagogical observations systematically and analyze the children's behaviour in order to get to know intellectual possibilities and developmental needs of every child. What is more, at the beginning of the year preceding the year the child starts his education in his first class of primary school, the teachers have to carry out an analysis about the child's school readiness so that there was still some time to take action to compensate and support the child adequately to the diagnosed needs.

The aim of that diagnosis is to collect the information which can help:

- parents to get to know their child's readiness to start education at school and so that they could support the child according to the needs to acquire that readiness,

- nursery school teachers to organize and work out the individual program to support the child's development which is to be executed in the year preceding the possible beginning of school education in the period from January to May,

- the lawyers from psychological and pedagogical counselling services where the child is to be sent when the nursery school teacher notices the need for the deeper diagnosis connected with some kind of specific educational needs of the individual child.

On the basis of that kind of diagnosis the nursery school teachers work out individual programs regarding the support and correcting the development of the child who needs it. In accordance with these programs there are supposed to be executed the didactic-compensatory classes. The diagnosis of the school readiness is also supposed to help the parents to get to know their child's readiness to start education at primary school. Thanks to that they can support their child's development both with the nursery school teacher. It can also help them decide whether to postpone their child's school education in case it was necessary. The analysis of the school readiness should be carried out by the teachers in the place which the children and their parents know well so at nursery schools, nursery school units at primary schools or in other forms of nursery school education. The recommendations concerning that matter which are included in the programs basis state that it is not only the birth certificate which should be taken into consideration while deciding if the child was ready to start his primary school education or not, but also his intellectual level. The children whose development takes place a bit more slowly should have time and should be given a chance to achieve the school 
readiness and it should not be as complicated as it has been so far.

In order to test the school readiness in Poland there is the School Readiness Scale.

The School Readiness Scale (SRS) is the observation method for the teachers which has the character of the assessment scale. It was worked out on the basis of the broadly defined school readiness connected with the cognitive, physical, emotional and social development of a child.

In the Scale there were listed those behaviors and abilities which are important while getting to know the achievements, preferences, interests and also difficulties experienced by the children due to attaining the school readiness. It is assumed that the school readiness of a child should be taken into consideration throughout his activeness in the preschool or school environment.

The School Readiness Scale (SRS) was worked out on the basis of widely understood school readiness connected with cognitive, physical, emotional and social development of the child. The Scale of the School Readiness consists of five parts (from A to E). In part (A) there are listed some ways of behaviour and abilities connected with the child's cognitive activity. Part (B) applies to the child's behaviour in his peer group. In part $(\mathrm{C})$ the teacher's attention was focused on the manifested independence of the children and their ability to cope with difficult situations. Part (D) of the scale concerns the child's task activity which is taken up individually or during the classes with the teacher's guidance. Part (E) of that method applies to the child's preparation to be able to read and write and to learn mathematics.

In each part there are the following subscales (in brackets there is the number of items):

1. School Abilities (20),

2. Cognitive Competences (12),

3. Motor Abilities (8)

4. Independence (12),

5. Non-Confrontational Features (12),

6. Social Activity (9).

These guidelines include complete descriptions of the fonts, spacing, and related information for producing your proceedings manuscripts. Please follow them.

\section{School Abilities}

The School Abilities subscale includes the items placed in part (A) and (E) of the School Readiness Scale (SRS). The essence of behaviour and abilities which are included in the given subscale have no homogeneous theoretical interpretation. They respond to the tasks given to the children during their nursery school education and at the same time to the teachers' expectations. The examples of these kind of tasks are: observing the cyclic changes in nature, the activities connected with counting, describing pictures, picture stories, practicing phonological skills, copying patterns. The behaviour and abilities are connected with various aspects of the children's development: attention, conceptual representation (numbers, space, time) and actions connected with it, operational and cause and effect thinking, psychomotor development.

The School Abilities subscale is created by the following behaviours and skills:

- a child is able to concentrate on the given activity

- A10 can compare the two pictures with some distinctive details

- A11 listens carefully, does not distract

- A12 distracts easily (U!)

- a child understands cause and effect connections between events

- E1 can describe the picture story

- E2 in the story he can include the cause and effect connections

- a child understands the relations and concepts connected with space

- A2 distinguishes the left-right directions

- A3 understands the concepts connected with space e.g. above, under, behind, besides

- A4 shows the directions on the sheet of paper e.g. up, down, left, right

- a child knows the concepts connected with the time category

- A5 knows the seasons of the year and phenomena connected with them

- A6 can say what the day of the week is and what the following day is

- a child can do the logical and mathematical operations

- A14 can put the new object in the already existing row

- E15 knows and uses the ordinal numbers

- E16 adds and subtracts the objects, tally clerks

- a child can do the activities connected with the language material

- E3 divides the sentence into words

- E4 divides the words into syllables and joins the syllables into words

- E5 has the ability of analysis and synthesis of the phonemes

- a child tries to read on his own (E6)

- a child does the graphomotor tasks

- E11does the jigsaw puzzle

- E13 copies and draws the patterns and easy geometrical figures

- E14 draws the patterns which resemble letters

\section{Cognitive Competences}

The Cognitive Competences subscale was created out of part (A) an (E) SRS and covers the behaviour 
and skills of the six years old child which are connected with his interests and cognitive achievements. They are: searching for and gathering experiences, linking them in more general cognitive categories (e.g. concepts), finding out some relationships between experiences and communicating these experiences with others. Both the children's abilities and the observatory indexes of these abilities are connected with various fields of the child's activities: with getting to know the world of nature, the social and symbolical worlds.

The Cognitive Competences consist of the following behaviours and abilities and related to them positions out of the SRS:

- a child displays the cognitive interest notices the relations between events and behaviours - A1 tries to explain the observed phenomena - A7 predicts the behaviour of other children

- A13 defines the names by applying them to more general categories

- E18 chooses number games while playing

- E19 is eager to solve mathematical riddles

- a child has the knowledge that goes beyond the direct experience (A15)

- a child expresses the elements of the selfknowledge and attempts of self-esteem

- A8 tells a lot about himself e.g. about what he likes doing

- A9 can tell the facts about what he knows and what he can do

- a child is able to read and has mathematical skills beyond expectations

- E7 has the abilities to read beyond the expected ones

- E17 adds and subtracts in memory

- E20 counts to 100 in memory

- E21 has mathematical abilities beyond the expected ones

\section{Motor Abilities}

The Motor Abilities subscale was created out of the positions placed in (B) and (E) SRS and applies to the child's activity and motor ability, physical coordination and manual dexterity and also to the child's satisfaction while having physical classes:

- a child likes physical activity

- B4 likes gymnastics and sports classes

- E8 likes handmade works

- a child has a good motor coordination

- B5 can catch and throw the ball easily

- B6 is able to ride a bike/ climbs the ladders

- B7 goes over the 'balance beam'

- a child has a manual dexterity

- E9 builds out of small building blocks

- E10 models some figures out of the Plasticine (e.g. people, animals)
- E12 can hold the pencil properly (the grip, muscle tension)

\section{Independence}

The Independence subscale was formed out of the positions placed in parts (C) and (D) SRS. The subscale covers the behaviour and the children's abilities which depend on resourcefulness, searching for the solution in difficult situations independently, trying to be independent. In this subscale there are included the actions which show that a child executes the purposeful forms of action, e.g. trying to finish the task. The essence of this subscale presents the character features such as resistance and persistence. Five positions in this scale are connected with the lack of independence which is manifested by being dependent, by asking for help frequently or resigning to finish the task on the child's own initiative. If these behaviours are not just transitory, they can signify that the given child has got some difficulties.

The following actions display the child's readiness to take independent actions and to work in group with the teacher's guidance:

- a child can do everyday activities independently

- C1 is able to get dressed (ties his shoelaces, buttons up or zips up his clothes)

- D6 can remember and do the task

- a child tries to overcome the difficulties on his own

- C2 takes independent actions to overcome the difficulties

- C3 is resistant while making attempts, does not become disaffected when it comes to misfortunes

- C4 initiates actions and tries to do the task in various ways

- a child takes the task-oriented and purposeful forms of action

- D1 is interested in the result and tries to finish his work

- D2 likes doing his works well and improves them

- a child avoids the situations which require independence - C5 they ask for help an adult too easily (U!)

- C6 imitates other children's behaviour and actions (U!)

- C7 avoids situations and tasks which require independence (U!)

- C8 tries to be close to the teacher or another adult person (U!)

- D5 asks for additional explanations and information (U!) 


\section{Non-Confrontational Features}

The Non-Confrontational Features subscale was mainly done out of part (B) SRS but it also includes one position from part (D) which shows that there are some contrary behaviours towards an adult. This subscale includes the positions which apply to social abilities: cooperating with the group, fulfilling some agreements, taking into consideration other children's rights, expressing negative feelings without hurting others. The children's behaviours listed here concern the child's attempts to solve conflicts and aspirations for being able to bring violent emotions under control in difficult situations. Seven positions of this subscale concern the lack of non-confrontational features. They display aggressive behaviour, violent emotions and evoking conflicts.

The child's non-confrontational features in SRS are made up of the following behaviours and abilities with their appropriate positions from the scale:

- a child behaves in accordance with the agreement

- B8 clears the toys and study aids

- B9 remembers about the rules of how to behave safely in the group

- B16 takes into consideration other people's rights, e.g. waits for his turn

- B17 requires to remind him about the agreement (U!)

- a child tries to solve the conflict situations

- B13 avoids the conflict situations

- B14 tries to cope with them in a peaceful manner

- B15 tells on other children, in the conflict situation he blames others (U!)

- a child displays strong emotions in difficult situations

- B18 often evokes conflicts (U!)

- B19 reacts with anger, is offended, turns away, goes away (U!)

- B20 gets angry easily, starts crying (U!)

- B21 is aggressive towards other children (U!)

- D7 objects, does not fulfill commands (U!)

\section{Social Activeness}

The Social Activeness subscale was created out of the positions placed in part (B) and (D) SRS. It applies to the child's behaviour and abilities which are connected with being with contacts and communicating with peers. There belong: the ability to communicate, ask questions, get information, inviting other children to play together, expressing themselves, being understood by others. This subscale covers the behaviour connected with the community like offering support and help and also the behaviour which shows that the children are able to express their feelings easily.

The following children's behaviours and abilities both with their SRS positions make up the Social Activeness:

- a child initiates the contacts with his peers

- B1 tries to contact other children in the way they can understand it

- B3 invites other children to talk and play together

- a child shows compassion and his willingness to help others

- B10 helps other children, tries to console others

- B11 can feel other children's feelings (can identify and name them)

- B12 tries to defend other children

- a child talks about himself eagerly and expresses his feelings

- B2 talks about important for him matters

- D3 shows happiness when it comes to the achieved results

- a child talks to his peers and the teacher with ease

- D4 often asks questions

- D5 asks for additional explanations and information

\section{Organization of observation}

The teacher's task is to observe whether and to what extent the given child manifests the behaviours and abilities listed in SRS and after the time spent on observations all that should be written down on the record sheet. There are the numbers from 1 to 4 to the assigned levels of manifested by the child types of behaviours and abilities which are used during the quantitative analysis of the results:

- yes (1) means that the given behaviour or ability is definitely manifested by the child,

- rather yes (2) means that the teacher could observe the given behaviour or ability but it was not rooted,

- rather not (3) means that the given behaviour or ability is very rare,

- no (4) means that the given behaviour or ability was not noticed by the teacher.

The (U!) sign means that it is a position with negative score.

In the subscales Cognitive Competences, Independence, Non-Confrontational Features, Social Activeness there is made an appraisal when it comes to the level of the school readiness with the use of the three descriptive categories:

- low score - there is the low level of the school readiness -1

- middle score - there is the medium level of the school readiness -2 
- high score - there is the high level of the school readiness -3

In the subscales School Abilities and Motor Abilities the analysis of the school readiness is carried out with the use of two descriptive categories:

- the results worse than the expected ones - the lower than expected level of the school readiness $-\mathrm{N}$

- the results in accordance with the expected ones - the results compatible with the expected level of the school readiness $-\mathrm{Z}$

The observation should be carried out for two weeks. During that time the teacher should observe not more than 10 children who participate in the classes with the whole nursery school group. The teachers should use SRS twice during the obligatory nursery school year:

- the preliminary observation carried out by the teacher in autumn

- the final observation carried out in April.

The aim of the initial observation is to help the teacher answer to the question what will make it easier and what more difficult for a child to learn at school. The results of the observation will then have the qualitative character. By registering the child's behavior and abilities and thanks to the qualitative analysis the teacher will be able to plan the way of work with a child and the whole group. It will also be helpful regarding the repeat observation to assess the changes of the children's behavior and their progress while acquiring abilities. Thus, the teacher should take into consideration the fact that there are some abilities e.g. connected with operational thinking, phonologic awareness or independence, which can come out as late as at the end of the nursery school education period.

The aim to observe the children's school readiness by the teacher is to determine to what extent a child is ready to start his school education regarding individual types of abilities and behaviors which can be put together in a form of the SRS and which are distinguished as the subscales. The information about the level of the school readiness, both with the qualitative analysis when it comes to the results of the observation, is important for parents and teachers while taking decisions connected with the child's education and handed over to the teachers of the first class of primary school (with parents' approval) which will make it easier to plan their work while taking into consideration the given educational needs of a child.

Before applying the method the teacher should collect the needed materials. In order to carry out the observation there are needed the following materials and helpful resources:

- paper for drawing A4,

- soft Plasticine (6 colors),
- pencils HB,

- crayons (12 colors),

- brushes for painting (thick ones and medium ones),

- poster paints (6 colors),

- covers on pencils (to handle them properly).

The objects listed above constitute the standard equipment of the unit when it comes to materials useful during art classes and graphomotor tasks. Thanks to the covers on pencils the teacher can check whether the observed difficulties undergo the correction. Moreover, in order to observe the motor and manual ability and also the visual-motor coordination among children, there are needed:

- three balls - each one of a different size (the diameter of the balls: from $7 \mathrm{~cm}$ to around 20 $\mathrm{cm}$ ),

- stamps (it would be favorable to have 3 sets) in the shape of the letters of the alphabet, the shape of numbers and in the shape of geometrical figures or animals,

- small building blocks like Lego, the plastic ones, 200 pieces, packed into one bucket or in a box,

- jigsaw puzzles (realistic pictures which depict situations of play and from everyday life, animals but no cartoons):

- 1 set /30-40 elements, the size of a picture A4, - 1 set/50-60 elements, the size of a picture A4,

- 1 set/70-100 elements, the size of a picture A4.

\section{Calculation results of school readiness}

Counting the results of the School Readiness Scale concerns the observation which takes place at the end of the compulsory nursery school year, whereas the results of the initial observation have only the qualitative character. In order to count the results of the SRS the teacher should:

- mark the appropriate answer at the blanket of the SRS form,

- write down in the summary table Summary of the Results of the Observation the points which correspond to the appropriate answers (yes -1 point, rather yes -2 points, rather not -3 points, no -4 points),

- count the severe results for each subscale by adding the points,

- count the counted results by subtracting the severe result from the given constant,

- determine the level of the school readiness of a given child in each subscale.

The counted result can be received by subtracting the severe result from the established numeric value. In the table there are presented numeric values from which there should be subtracted the severe results. 


\begin{tabular}{|c|c|c|}
\hline \multirow{2}{*}{ School Abilities } & Severe Results & Counted Results \\
\cline { 2 - 3 } & S1 & $75-\mathrm{S} 1$ \\
\hline \multirow{2}{*}{ Cognitive Competences } & & \\
\cline { 2 - 3 } & $\mathrm{S} 2$ & $48-\mathrm{S} 2$ \\
\hline \multirow{2}{*}{ Motor Efficiency } & $\mathrm{S} 3$ & $32-\mathrm{S} 3$ \\
\cline { 2 - 3 } Independence & $\mathrm{S} 4$ & $23-\mathrm{S} 4$ \\
\cline { 2 - 3 } & & \\
\hline \multirow{2}{*}{ Non-Conflict Nature } & $\mathrm{S} 5$ & 13-S5 \\
\cline { 2 - 3 } & & \\
\hline \multirow{2}{*}{ Social Activeness } & $\mathrm{S} 6$ & $36-\mathrm{S} 6$ \\
\cline { 2 - 3 } & & \multicolumn{2}{|c}{} \\
\hline
\end{tabular}

The teacher can count the results of the School Readiness Scale on his own or fill in the observation blanks on his computer in the program enclosed on a $\mathrm{CD}$, which can count the results.

The ranges of results counted for the School Abilities subscale (0-60) (two levels of the school readiness).

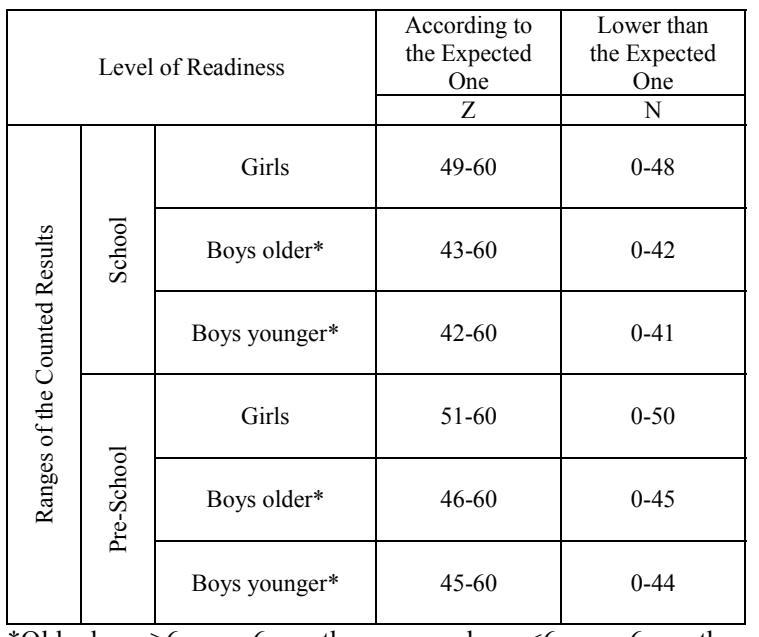

While determining the level of the school readiness of a child in the subscale School Abilities (tab. 28), there should be taken into consideration the institution the child goes to, the gender and when it comes to boys also their age. The counted result should be found in the table while taking into consideration the criteria above. The example: If a boy who is 6 years and 6 months old or more goes to the pre-school department at primary school and he gets the counted result of 50 points in the subscale School Abilities, which can be found in the range 4360 points, then his level of school readiness is according to the expected one. If a girl who goes to pre-school gets the same result of 50 points in the subscale School Abilities then her level of the school readiness is lover that expected.

The ranges of the counted results for the subscale Motor Efficiency (0-24) (two levels of the school readiness).

\begin{tabular}{|c|c|c|c|}
\hline \multicolumn{2}{|c|}{ Level of Readiness } & \multicolumn{2}{|c|}{ Ranges of the Counted Results } \\
\cline { 3 - 4 } & Girls & Boys \\
\hline $\begin{array}{c}\text { According to the } \\
\text { Expected One }\end{array}$ & $\mathrm{Z}$ & $21-24$ & $20-24$ \\
\hline $\begin{array}{c}\text { Lower than the } \\
\text { Expected One }\end{array}$ & $\mathrm{N}$ & $0-20$ & $0-19$ \\
\hline
\end{tabular}

While determining the child's the level of the school readiness in the subscale Motor Efficiency there should be taken into consideration only the gender of a child. The counted result should be found in the table considering the mentioned criterion. The example: the result of 20 counted points in the subscale Motor Efficiency when it comes to a boy gives the level of the school readiness in accordance with the expected one. The result of 20 counted points in the in the subscale Motor Efficiency regarding a girl gives the level of the school readiness lower than expected.

The ranges of the counted results for the subscale Cognitive Competences (0-36) (three levels of the school readiness).

\begin{tabular}{|c|c|c|c|c|c|}
\hline \multirow{2}{*}{$\begin{array}{c}\text { Level of } \\
\text { Readiness }\end{array}$} & \multicolumn{3}{|c|}{ Ranges of the Counted Results } \\
\cline { 3 - 6 } & \multicolumn{2}{|c|}{ School } & \multicolumn{2}{c|}{ Pre-School } \\
\cline { 3 - 6 } & Girls & Boys & Girls & Boys \\
\hline High & 3 & $25-36$ & $25-36$ & $26-36$ & $27-36$ \\
\hline Medium & 2 & $10-24$ & $9-24$ & $12-25$ & $11-26$ \\
\hline Low & 1 & $0-9$ & $0-8$ & $0-11$ & $0-10$ \\
\hline
\end{tabular}

While determining the child's the level of the school readiness in the subscale Cognitive Competences there should be taken into consideration the department the child goes to and the gender. The counted result should be found in the table considering the mentioned criteria. The example: the result of 25 counted points in the subscale Cognitive Competences when it comes to a girl from the pre-school department at primary school gives the high level of the school readiness and the same result in the subscale Cognitive Competences achieved by a girl from pre-school gives the medium level of the school readiness.

The ranges of the counted results for the subscale Independence (0-36) (three levels of the school readiness).

While determining the child's the level of the school readiness in the subscale Independence there should be taken into consideration the department the child goes to, the gender and regarding boys also their age. The counted result should be found in the table considering the mentioned criteria. The example: If a boy who is less than 6 years 6 months old and goes to the pre-school department at primary school gets the result of 32 counted points in the subscale Independence then his level of the school readiness is high. If a girl who goes to pre-school gets 32 points in the subscale Independence then the level of her school readiness is medium. 


\begin{tabular}{|c|c|c|c|c|c|}
\hline \multicolumn{3}{|c|}{ Level of readiness } & High & Medium & Low \\
\hline \multirow{6}{*}{ 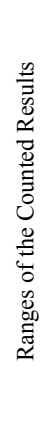 } & \multirow{3}{*}{$\begin{array}{l}\overrightarrow{0} \\
\overline{0} \\
\dot{c}\end{array}$} & Girls & $34-36$ & $20-33$ & $0-19$ \\
\hline & & Boys older* & $33-36$ & $16-32$ & $0-15$ \\
\hline & & $\begin{array}{c}\text { Boys } \\
\text { younger* }\end{array}$ & $32-36$ & $15-31$ & $0-14$ \\
\hline & \multirow{3}{*}{ 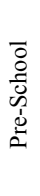 } & Girls & $35-36$ & $22-34$ & $0-21$ \\
\hline & & Boys older* & $34-36$ & $17-33$ & $0-16$ \\
\hline & & $\begin{array}{c}\text { Boys } \\
\text { younger* }\end{array}$ & $33-36$ & $16-32$ & $0-15$ \\
\hline
\end{tabular}

The ranges of the counted results for the subscale Non-Conflict Nature (0-36) (three levels of the school readiness).

\begin{tabular}{|c|c|c|c|}
\hline \multicolumn{2}{|c|}{ Level of Readiness } & \multicolumn{2}{c|}{ Ranges of the Counted Results } \\
\cline { 3 - 4 } & & Girls & Boys \\
\hline High & 3 & 36 & $35-36$ \\
\hline Medium & 2 & $25-35$ & $19-34$ \\
\hline Low & 1 & $0-24$ & $0-18$ \\
\hline *Older boys $>6$ years 6 months; younger boys $<6$ years 6 months.
\end{tabular}

While determining the child's the level of the school readiness in the subscale Non-Conflict Nature there should be taken into consideration only the gender of a child. The counted result should be found in the table considering the mentioned criterion. The example: 19 counted points in the subscale NonConflict means that there is a low level of the school readiness regarding a girl and a medium level of the school readiness concerning a boy.

The ranges of the counted results for the subscale Social Activeness (0-27) (three levels of the school readiness).

\begin{tabular}{|c|c|c|c|c|}
\hline \multirow{2}{*}{\begin{tabular}{c}
\multirow{2}{*}{$\begin{array}{c}\text { Level of } \\
\text { Readiness }\end{array}$} \\
\cline { 3 - 5 }
\end{tabular}} & \multicolumn{3}{|c|}{ Girls } & \multicolumn{2}{|c|}{ Boys } \\
\cline { 3 - 5 } & & $25-27$ & $24-27$ & $25-27$ \\
\hline High & 3 & $17-24$ & $14-23$ & $15-24$ \\
\hline Medium & 2 & $17-24$ & $0-14$ \\
\hline Low & 1 & $0-16$ & $0-13$ & \multicolumn{2}{c}{} \\
\hline
\end{tabular}

While determining the child's the level of the school readiness in the subscale Social Activeness there should be taken into consideration the gender of a child and when it comes to a boy also the educational department the child goes to. The counted result should be found in the table considering the mentioned criteria. The example: If a boy from the pre-school department at primary school gets the result of 24 counted points in the subscale Social Activeness then his level of the school readiness is high, whereas his friend from preschool who gets 24 counted points in the subscale Social Activeness has the medium level of the school readiness.

Each child who achieves the low counted result and low or lower than expected level of the school readiness in the given range needs the deeper diagnose so that it was possible to answer to the question how to support his development and further education. The low level of readiness or incompatible with expectations points out the risk of failure. If a teacher went only as far as to assess the School Abilities, he would not see the risk of failure connected with low level of school readiness in other aspects of the child's development. He would also not notice the stronger sides of the child's development and omit them while planning work. Children with motor or mental disability determined by developmental malfunctions require the deeper diagnose. It also concerns the children who have persistent difficulties with acquiring social abilities.

The teacher's decisions, when it comes to the children who get lower results in subscales of the SRS, will depend on the moment the observation is carried out. If the observation takes place at the beginning of the second semester, the teacher's decision will concern working out and executing the plan of how to support the child's development. The teacher will also take into consideration the consultations with other teachers at school or preschool and with specialists - a psychologist, a speech therapist, a psychotherapist and a physiotherapist. The results achieved thanks to one method alone, loaded by - as all observation methods - mistakes typical of human perceiving, cannot be the only one basis to make pedagogical decisions.

\section{Conclusion}

The school readiness scale is in conformity with the teachers' pedagogical practice regarding the preschool education - it was verified thanks to the pilot and standard studies. It includes those behaviors and abilities of the children which are on the one side mentioned by the developmental psychologists as the achievements of the pre-school age and on the other hand - they are important while perceiving children by the teachers. This method brings in to the school readiness assessment the social abilities and the children's independence in a wider range than ever. There is a conviction that the children's experiences, their way of communicating to others and expressing emotions, undertaking new forms of activeness and coping with difficult situations have a big influence on their preparation to school education. In the school readiness assessment there were also included some cognitive achievements, which cannot be expected from all of the children. Thus, the term of readiness also relates to those children whose cognitive competences are high, which should be taken into consideration in the program and in the teacher's methods of work. The SRS is the method which requires keeping the appropriate conditions and procedures. The time of observation, the needed materials and helping tools, the number of children 
that can be observed at a time are determined precisely in this method. The procedure of using the SRS requires the initial observation and to plan and execute by the teacher all the educational activities which support the child's development and the learning process. The observation at the end of the obligatory pre-school year makes the summary of the teacher's work.

The important element of the procedure when using the SRS is the cooperation with parents of the children. Parents should get information from the teacher about the method and about the results of their child's observation. In everyday life there are many situations which can be used by parents consciously to help their children achieve the better school readiness.

\section{References}

[1] Frydrychowicz, A., E. Koźniewska, A. Matuszewski, and E. Zwierzyńska, Skala gotowości szkolnej. Podręcznik, Centrum Metodyczne Pomocy Psychologiczno-Pedagogicznej, Warszawa, 2006

[2] Klim-Klimaszewska, A., Pedagogika przedszkolna. Nowa podstawa programowa, Wyd. Erica, Warszawa, 2010

[3] Koźniewska, E., A. Matuszewski, and E. Zwierzyńska, Skala Gotowości Edukacyjnej Pięciolatków (SGE-5). Obserwacyjna metoda dla nauczycieli, Ośrodek Rozwoju Edukacji, Warszawa, 2010

[4] Podhajecka, M., Kompetencie v predskolskej edukacii, Wyd. PU, Presov, 2012 